\title{
Current treatment of multidrug resistant tuberculosis in Ethiopia: an aggregated and individual patients' data analysis for outcome and effectiveness of the current regimens
}

\author{
Setegn Eshetie ${ }^{1 *}$ (D) Animut Alebel ${ }^{2}$, Fasil Wagnew ${ }^{2}$, Demeke Geremew ${ }^{3}$, Alebachew Fasil $^{4}$ and Ulrich Sack ${ }^{5}$
}

\begin{abstract}
Background: The programmatic management of Multidrug-resistant tuberculosis (MDR-TB) is entirely based on a WHO recommended long-term, 18-24 month lasting treatment regimen. However, growing evidence shows that low treatment success rate and high rates of adverse events are associated with this regimen. Up to date, the MDR-TB treatment outcome is not sufficiently understood in Ethiopia. Therefore, this analysis aimed to determine the pooled estimates of successful (cure, completed, or both), and poor outcomes (death, failure, and lost to follow ups).
\end{abstract}

Method: A systematic search was performed to identify eligible studies reporting MDR-TB treatment outcomes in Ethiopia. Relevant studies for our analysis were retrieved from PubMed database search, Google Scholar and institutional repository sites of Ethiopian universities up to March 15, 2018. The primary outcome was treatment success, referring to a composite of cure and treatment completion. A random effect model was used to calculate pooled estimates.

Results: Six studies reporting treatment outcome on the 1993 MDR-TB patients were included in this analysis. Of the cases, the 1288 and 442 patients had a successful and poor outcome, respectively. In the pooled analysis, treatment success was observed in $59.2 \%(95 \% \mathrm{Cl}, 48.1-70.4)$ of patients, while $23.3 \%(95 \% \mathrm{Cl}, 19.7-27.0 \%)$ of patients had a poor outcome. in sub-group analysis, $46.1 \%(95 \% \mathrm{Cl}, 34.2-58.0)$ were cured, $12.8 \%(5.7-20.0)$ treatment completed, $14.3 \%$ (11. 5-17.2) died, 7.5\% (3.7-11.3) lost to follow up, and 1.6\% (1.1-2.2\%) experienced treatment failure. The 25.0\% (14.6-35.5) patients whose treatment outcome was not assessed (on treatment or transfer-out).

Conclusion: The result of this study highlight treatment success among MDR-TB is below acceptable range. To update the current treatment regimen, the levels of evidence need to be replicated through meticulous surveillance systems.

Trial registration: Study protocol registration: CRD42018090711.

Keywords: Ethiopia, MDR-TB, Treatment success

\footnotetext{
* Correspondence: wolet03.2004@gmail.com

${ }^{1}$ Department of Medical Microbiology, School of Biomedical and Laboratory

Sciences, College of Medicine and Health Sciences, University of Gondar,

Gondar, Ethiopia

Full list of author information is available at the end of the article
}

(c) The Author(s). 2018 Open Access This article is distributed under the terms of the Creative Commons Attribution 4.0 International License (http://creativecommons.org/licenses/by/4.0/), which permits unrestricted use, distribution, and reproduction in any medium, provided you give appropriate credit to the original author(s) and the source, provide a link to the Creative Commons license, and indicate if changes were made. The Creative Commons Public Domain Dedication waiver (http://creativecommons.org/publicdomain/zero/1.0/) applies to the data made available in this article, unless otherwise stated. 


\section{Background}

Multidrug-resistant tuberculosis (MDR-TB) is defined by resistance to at least the two most powerful first line anti-tuberculosis drugs, isoniazid, and rifampicin [1]. MDR-TB remains a major public health crisis, with an estimated 490, 0000 new cases are emerging globally each year [2]. The increasing incidence of drug resistant TB is mainly reported from resource-limited countries where TB care programs are compromised. Globally, $3.9 \%(95 \%$ CI $2.7-5.1 \%)$ of new cases and $21 \%$ (95\% CI $15-28 \%)$ of previously treated $\mathrm{TB}$ cases were in MDR-TB cases. It is also estimated that each year 580,000 and 250,000 deaths reported among new and previously treated MDR-TB cases [3], respectively. According to the recent global report, 2900 (1800-4000) MDR-TB cases were estimated among notified pulmonary TB in Ethiopia [2]. A latest meta-analysis study also reported that $2 \%$ of new cases and $17 \%$ among previously treated cases were MDR-TB patients [4].

Programmatic management of drug-resistant TB treatment is based on conventional or longer MDR/ rifampicin resistant (RR)-TB treatment regimens, which lasts for 20-24 months and achieved a success rate nearly in $50 \%$ of patients, worldwide [5]. The low successful treatment outcome might be due to prolonged treatment period, low drug efficacy and toxic regiments. Recent advances show that high treatment success was achieved by using a short course treatment regimen. To date, few studies have been conducted to reveal the effectiveness of short-course MDR/or RR-TB treatment regimen. The recent meta-analysis studies showed that the treatment success rate using a shorter MDR/RR-TB treatment regimen was ranged from $83.0-83.7 \%$ [6-8], which is significantly higher than a meta-analysis reported a success rate for conventional, longer MDR/RR-TB treatment regimen (54\%) [9]. Moreover, the effectiveness of shorter course MDR/RR-TB treatment was also explored in other institutional and observational studies. A study from nine African countries revealed that the treatment success and cure rate of short MDR/RR-TB regimen was $81.6 \%$ and $72.4 \%$, respectively [10]. Similarly, promising reports for short course MDR/RR-TB treatment were also noted in Bangladesh, Niger, and Cameron [11-14].

Generally, the existing facts asserted that the safety and effectiveness of the shorter MDR/RR-TB regimen are superior compared to the previously appreciated reports for the longer MDR/RR-TB regimen. Even more, a 9-12 month regimen for MDR/RR-TB was also recommended by World Health Organization (WHO) [15]. But, prior to putting this regimen in place or before this regimen can be prescribed for the MDR/RR-TB patients in a given country, the level evidence in both regimens needs to be replicated and supported through rigorous research and with a large number of patients, in order to conclude that this most effective treatment for MDR/ RR-TB. Besides, still findings are based on observational design, and therefore future researches need to be focus on high-level designs such as randomized controlled trials (RCTs) to generate highly relevant evidence in this regard. Most importantly, this short course treatment should not completely replace the conventional treatment approach since the fact that patients with fluoroquinolones or other 2nd line drug resistance, extra-pulmonary tuberculosis, pregnancy and with severe clinical problems are known not to be considered for shorter MDR/RR-TB regimen. In this case, the national TB control program (NTPs) in need to maintain the conventional treatment approaches.

The MDR-TB treatment in Ethiopia is mainly based on a standard or long course treatment, which is known as less effective to achieve high levels of successful outcome. The Ethiopian Federal Ministry of Health adopted a standardized regimen consists of an 8-month intensive phase with a combination of pyrazinamide, capreomycin, levofloxacin and prothionamide or ethionamide and cycloserine, a 12-month continuation phase with a combination of pyrazinamide, levofloxacin,prothionamide or ethionamide and cycloserine [16].

By far, few observational studies have been conducted to reveal treatment outcomes among MDR-TB patients, and even the findings are inconsistent and representing only local information. Hence, the effectiveness of the standard MDR-TB treatment was not fully assessed in Ethiopia. Therefore, we report here a meta-analysis of observational studies of regimens of 18-24 months in duration whose composition was based on a standardized longer term regimen. The purpose of the study was first, to determine the pooled successful and unfavorable treatment outcome measures. Secondly, the sub-group analysis was also done to estimate the proportion of cured and treatment completed cases among successfully treated cases, and to evaluate poor treatment outcomes including treatment failures, deaths, and lost to follow ups.

\section{Methods}

\section{Studies/setting}

Studies that reported the treatment outcome among MDR-TB patients in Ethiopia were included for this analysis. However, studies focused on non-MDR or drug-susceptible $\mathrm{TB}$ patients were not eligible for this study.

\section{Study protocol registration}

The study has been registered in PROSPERO database with protocol number, CRD42018090711. 


\section{Intervention}

The original studies reported an outcome from patients treated with a longer, $18-24$ months MDR-TB treatment regimen.

\section{Comparison}

MDR-TB treatment in Ethiopia is largely based on a standardized longer regimen. Therefore, no comparison was undertaken for this analysis.

\section{Outcomes}

MDR-TB treatment outcomes were evaluated as treatment success and unfavorable outcome. Successful outcomes described as patients meeting the definition of Cure or treatment completed. Poor treatment outcomes refer to patients meeting the definition of death, lost to follow up, and treatment failed.

\section{Search strategy and quality evaluation}

The PubMed database was employed to retrieve the available research reports in Ethiopia. A highly sensitive search strategy was developed using the combination of the following Keywords; treatment outcome AND multidrug-resistant tuberculosis OR drug-resistant tuberculosis OR MDR-TB AND Ethiopia, both as exploded as MESH headings and free-text terms. Manual searching was also done from the institutional repository websites of Ethiopian universities (such as Addis Ababa University, and University of Gondar) to include unpublished reports. Besides, grey literature searching was also performed using Google Scholar database to collect non-PubMed indexed articles. Electronic database searches were conducted in February and March 2018. Two authors (SE, AE) reviewed all abstracts and a full-text article, with the final decision, was made through consensus. In cases of disagreement, the consensus was achieved through arbitration of other authors.

Studies were potentially acceptable if they report the treatment outcome on at least 100 MDR-TB patients within a defined study design approach, retrospective, and prospective cohorts. The original studies were also rated as good quality when the average treatment duration was 12 months and above. However, treatment outcome other than MDR-TB was not examined in our meta-analysis. The overall quality score of the included studies was measured using the Newcastle-Ottawa quality assessment scale for observational studies (Additional file 2: Table S2).

\section{Data extraction and statistical analysis}

Two investigators (SE, AA) extracted data using a standard abstraction form. In the case of disagreement, other investigators (FW, US) took part to resolve the differences through discussion. Data abstraction was carried out with regard treatment outcomes. The primary outcome of this study was treatment success included cure, treatment completed, or both. The secondary outcome of the study was poor outcomes (such as, lost to follow up, death and failure). Moreover, for each included study the following information was also collected; HIV status, and previous history of anti-TB treatment.

The pooled proportions of the treatment outcomes with 95\%CI were statistically measured using Stata version 11.0 software (College Station, TX: StataCorp LP). A Freeman-Tukey-type arcsine square-root transformation and DerSimonian and Laird random-effects model were used to stabilize variances and calculate pooled estimates [17].The sensitivity analysis on primary outcome was done using a Bayesian random-effects model with Monte Carlo Markov chain simulations of variability [18]. The $\mathrm{I}^{2}$ statistic was used to assess the proportion of overall variation attributable to between-study heterogeneity [19].

\section{Results}

Selection process and description of the included studies

The selection process is depicted in Fig. 1, a total of 132 abstracts were identified from an initial electronic database search. Following title and abstract evaluation, 100 citations were excluded and the remaining 32 were subjected to full-text evaluation. After full-text review, 2 studies were found to be eligible for the analysis and a further 4 studies were obtained through manual searching, institutional repository sites of Addis Ababa University and email contact from the primary investigator. Finally, 6 studies were found to be acceptable for our analysis [20-25].

The baseline characteristics of the studies were indicated Table 1, of the included studies, three were retrospective cohorts, and the remaining were descriptive retrospective chart reviews and prospective cohort studies. All studies reported the patients' previous history of anti-TB drug treatment and HIV co-infection. The included studies reported treatment outcome among 1993 MDR-TB patients representing Northwestern, Northeastern, Central, Eastern and Southern Ethiopia, MDR-TB care centers. Of the patients, successful treatment outcome was observed in 1288 patients, while 442 were poorly treated cases (Table 2). However, the treatment outcome of 263 patients was not evaluated; this was due to either the transfer out or patients on the course of treatment. Relatively high treatment success rate was reported in Meressa et al. (78.6\%) [25] followed by Tolera et al. (65.9\%) [24] and Alene et al. (63.6\%) [22]. On the other hand, a high proportion of patients with poor outcome was observed in Tolera et al. (34.1\%) [24], followed by Alene et al. (25.6\%) [22] and Baye et al. (22.7\%) [20]. 


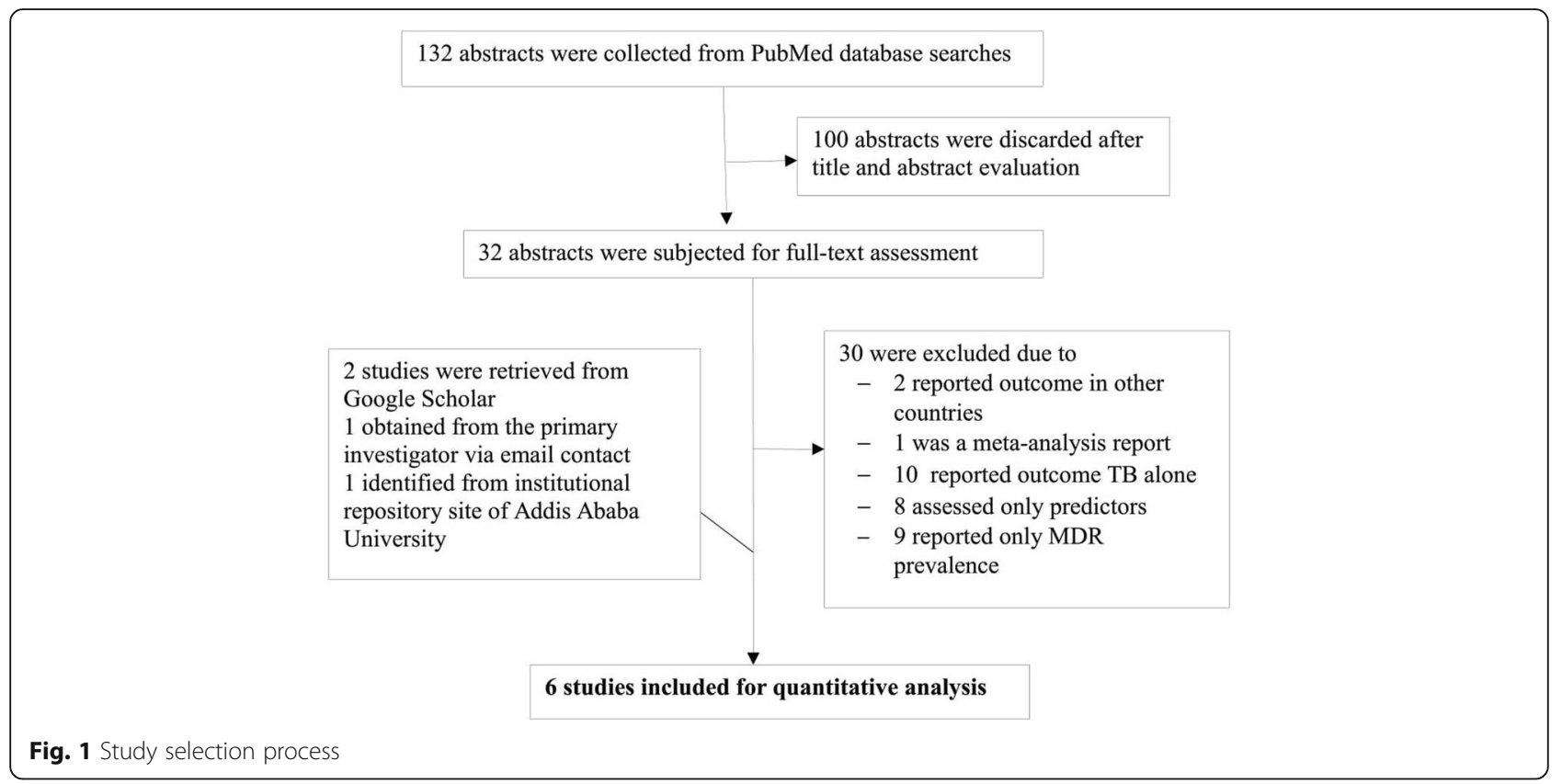

\section{MDR-TB treatment outcomes}

In the present analysis, the pooled treatment success was estimated as $59.2 \%(95 \% \mathrm{CI}, 48.1-70.4)$. The high heterogeneity between studies was observed $\left(\mathrm{I}^{2}, 96.3 \%\right)$ (Fig. 2), but evaluation of the publication bias is not recommended for a meta-analysis of lower than 10 studies. Likewise, the overall poor treatment outcome was also determined, as seen in Fig. 3, 23.3\% (95\%CI, 19.7$27.0 \%$ ) of the patients were unsuccessfully treated. High heterogeneity was also indicated $\left(\mathrm{I}^{2}, 70.7 \%\right)$.

In sub-group analysis, $46.1 \%(95 \% \mathrm{CI}, 34.2-58.0)$ and 12.8\% (95\% CI, 5.7-20.0) of MDR-TB patients were cured and treatment completed, respectively (Fig. 4). Moreover, the pooled proportion of poor treatment outcomes was also presented in Fig. 5, 14.3\% (95\%CI, 11.5-17.2) died, $7.5 \%(95 \% \mathrm{Cl}, 3.7-11.3)$ lost to follow up, and $1.6 \%$ $(95 \% \mathrm{Cl}, 1.1-2.2 \%)$ were patients with treatment failure. In Fig. 5, 25.0\% $(95 \% \mathrm{Cl}, 14.6-35.5)$ of the MDR-TB patients whose treatment outcome was not examined, as noted in the original studies, these were patients on treatment or transfer-out.

\section{Discussion}

Though the WHO task force set a strategic plan to target of $75 \%$ successful treatment outcome of MDR-TB by the end of 2015 in global bases [26], many countries including Ethiopia have not yet achieved this ambitious aim. Maximizing the favorable treatment outcome in MDR-TB is a global health priority and one of the key performance indicators of WHO's End TB strategy [27]. Ethiopia has made the treatment of MDR-TB a national health priority [28].
The national MDR-TB treatment program based on the recommendations from the 2011 update of Guidelines for the programmatic management of drug-resistant tuberculosis [29].The treatment of MDR-TB requires a long lasting drug exposure, and is also significantly associated with high rates of adverse drug events. Recent meta-analysis report documented a lower treatment success rate among patients treated with a longer MDR-TB regimen compared to those treated with a short course MDR-TB treatment [9].

To our knowledge, this is the first combined analysis to evaluate the effectiveness of the currently used MDR$\mathrm{TB}$ treatment program in Ethiopia. In the present analysis, the pooled estimate of successful treatment was 59.2\% (95\%CI, 48.1-70.4), while 23.3\% (95\%CI, $19.7-$ $27.0 \%)$ had poor treatment outcome. The treatment success observed in this analysis is comparable with the results of recently published meta-analyses of the outcomes of MDR-TB treatment with conventional drug regimens. Notably, a $54 \%$ treatment success rate for conventional MDR-TB treatment regimen was found in a meta-analysis conducted by Ahuja et al. [9]. Similar MDR treatment outcome was estimated in a meta-analysis conducted by Orenstein et al. [30], and pooled analysis from 21 countries [31], showed $62 \%$ successful outcome. Thus, it is suggested that the need to modify the duration and the composition of the current MDR-TB treatment regimens. Because of the lengthy therapy, toxicity and fewer efficacies of second-line anti-TB drugs resulting high rates of an unfavorable outcome subsequently lead to the rapid emergence of the extensively drug-resistant $\mathrm{TB}$. 


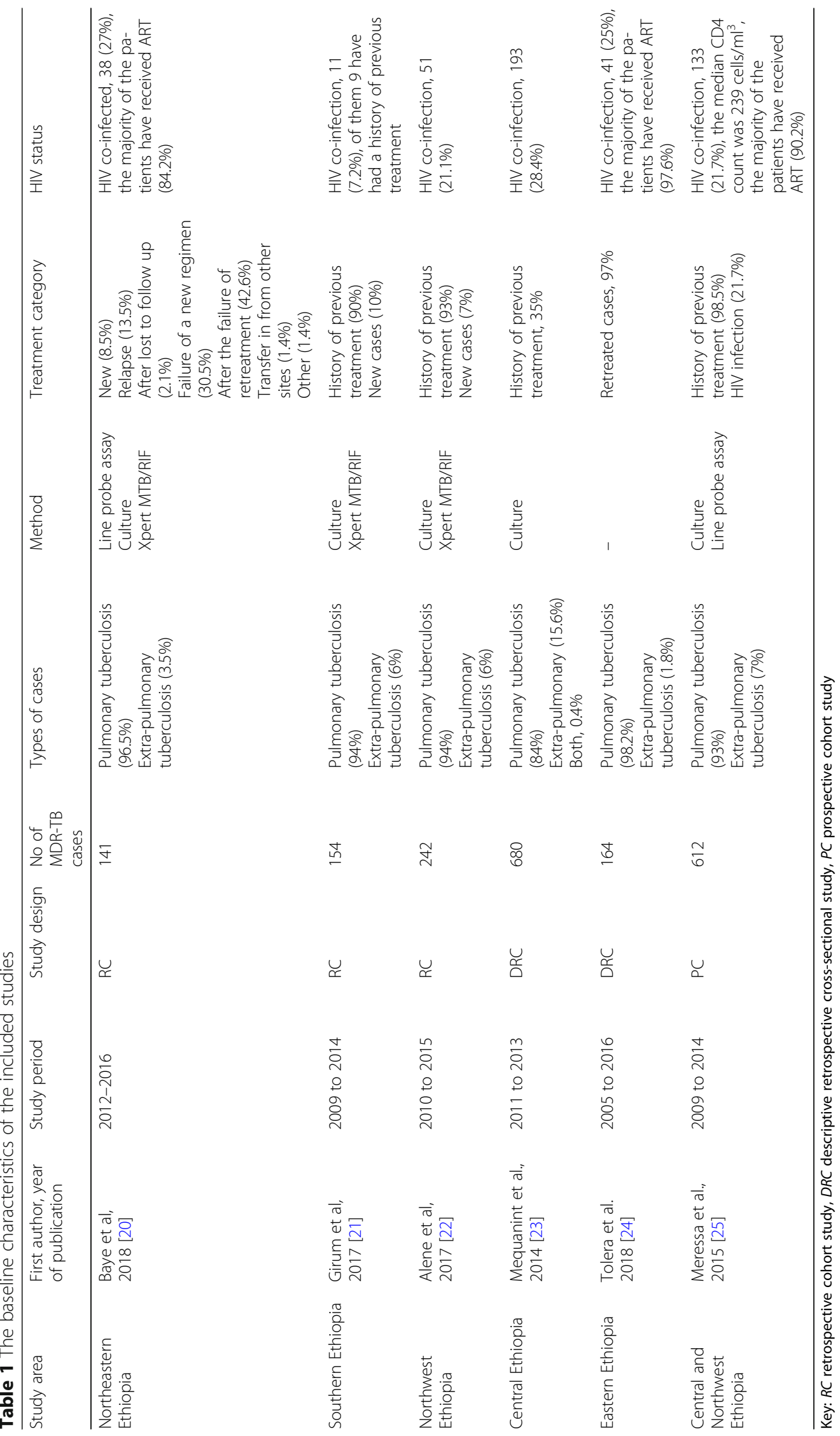




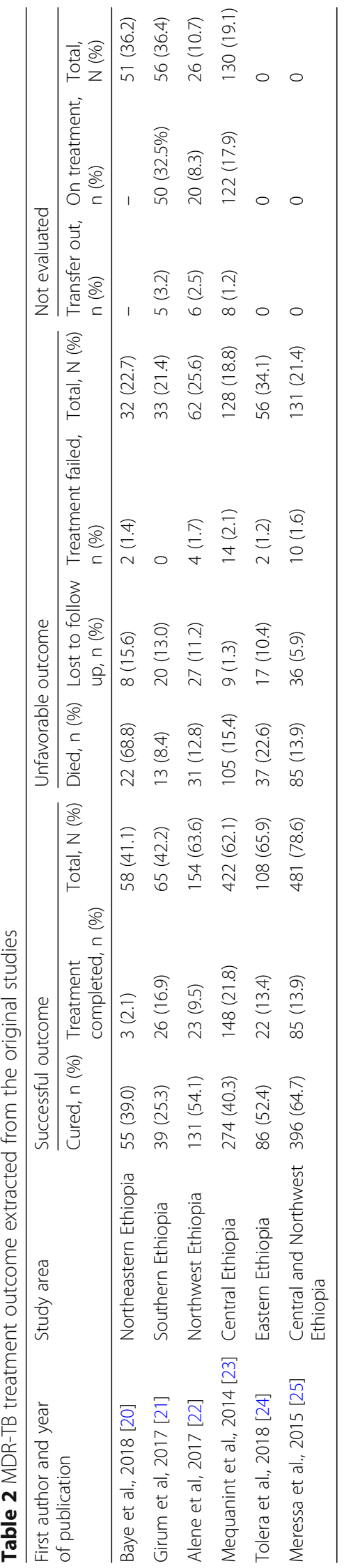




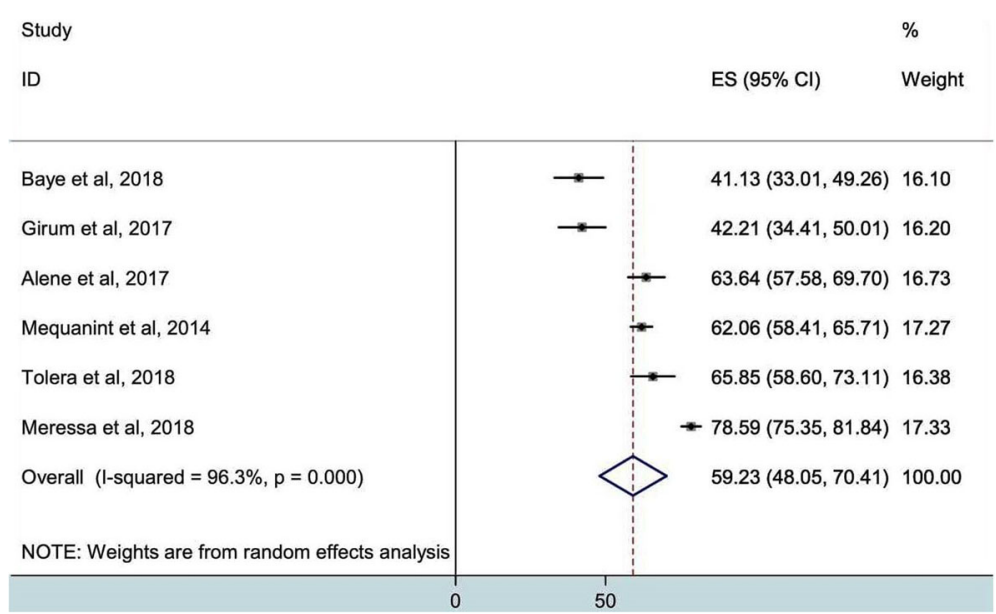

Fig. 2 Pooled estimates of successful treatment outcome

Most recently, an effective standardized treatment regimen lasting less than 12 months has been adopted in countries like Bangladesh, Benin, Burkina-Faso, Burundi, Cameroon, Central African Republic, Côte d'Ivoire, DR Congo, Guinea, Niger, Rwanda, Senegal, Swaziland, and Uzbekistan [10-14]. The regimen composed of the initial phase of 4 months therapy with kanamycin, moxifloxacin, prothionamide, clofazimine, isoniazid, pyrazinamide and ethambutol, and followed by 5 months of treatment with moxifloxacin, clofazimine, pyrazinamide, and ethambutol. Based on the data from recent studies, high treatment success has been achieved in above-mentioned countries. Most importantly, meta-analysis studies revealed that the treatment success rate using shorter MDR/RR-TB treatment regimens was ranged from $83.0-83.7 \%[6,7]$, which higher than previously appreciated treatment outcome. Furthermore, an observational survey of nine African countries also noted promising results of using 12-month regimen [10].

Of note, the latest advancement asserted that the effectiveness of the shorter treatment regimen, in May 2016 WHO moves one step forward to update drug-resistant TB treatment guideline, and underscored the recommendation on the use of the 9-12 month treatment regimen [15]. This encouraged the National Tuberculosis Program (NTP) of Ethiopia need to test and implement a similar 12-month regimen. Though there are several positives with this regimen, its use could be restricted by the fact that patients with fluoroquinolones or other 2nd line drug resistance, extra-pulmonary tuberculosis, pregnancy and with severe clinical problems are known not to be considered for shorter MDR/RR-TB regimen. In this case, the NTPs need to preserve the conventional treatment approaches.

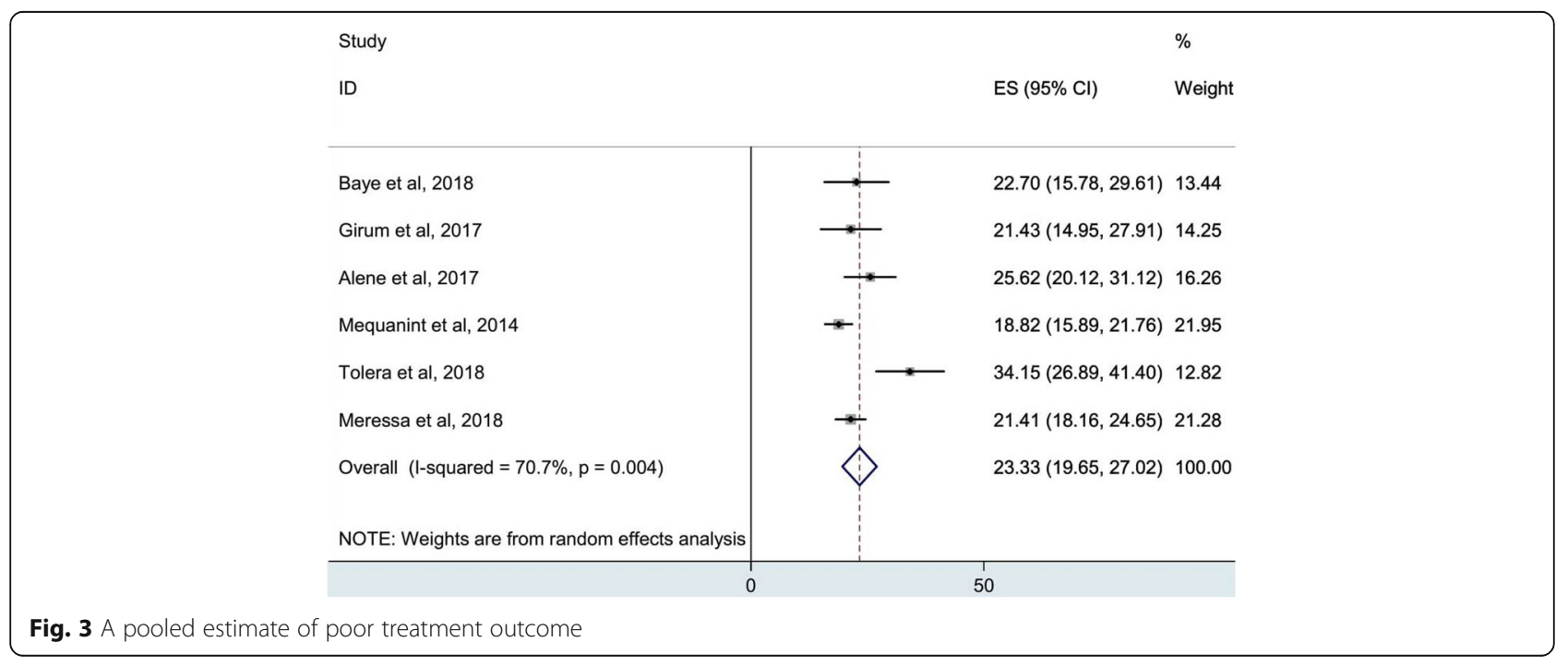




\begin{tabular}{|c|c|c|}
\hline \multicolumn{2}{|l|}{ Author } & ES $(95 \% \mathrm{Cl})$ \\
\hline Cured & & \\
\hline Baye et al, 2018 & & $39.01(30.96,47.06)$ \\
\hline Girum et al, 2017 & $\rightarrow$ & $25.32(18.46,32.19)$ \\
\hline Alene et al, 2017 & & $54.13(47.85,60.41)$ \\
\hline Mequanint et al, 2014 & & $40.29(36.61,43.98)$ \\
\hline Tolera et al, 2018 & & $\rightarrow \quad 52.44(44.80,60.08)$ \\
\hline Meressa et al, 2018 & & $\rightarrow 64.71(60.92,68.49)$ \\
\hline Subtotal (l-squared $=96.5 \%, p=0.000)$ & & $=\quad 46.09(34.17,58.01)$ \\
\hline & & \\
\hline Treatment completed & & \\
\hline Baye et al, 2018 & . & $2.13(0.53,3.73)$ \\
\hline Girum et al, 2017 & $\rightarrow$ & $16.88(10.97,22.80)$ \\
\hline Alene et al, 2017 & $*$ & $9.50(5.81,13.20)$ \\
\hline Mequanint et al, 2014 & $*$ & $21.76(18.66,24.87)$ \\
\hline Tolera et al, 2018 & $*$ & $13.41(8.20,18.63)$ \\
\hline Meressa et al, 2018 & + & $13.89(11.15,16.63)$ \\
\hline Subtotal (l-squared $=96.8 \%, p=0.000)$ & 8 & $12.84(5.67,20.01)$ \\
\hline
\end{tabular}

Fig. 4 The pooled proportion of cured and treatment completed cases

In sub-group analysis, it also estimated that $46.1 \%$ (34.2-58.0) cured, $12.8 \%(5.7-20.0)$ treatment completed, $14.3 \%$ (11.5-17.2) died, 7.5\% (3.7-11.3) lost to follow uped, $1.6 \%(1.1-2.2 \%)$ experienced treatment failure and $25.0 \%(14.6-35.5)$ of the patients whose treatment outcome was not evaluated. Particularly, the cure rate indicated in our analysis is substantially inferior to the results reported with a shorter MDR-TB regimen [15]. Aside from the drug-resistant nature of the infection, the low cure rate might be due to lack of sufficient MDR-TB care centers, poor patient adherence, the low performance of NTPs at central and regional levels, poor efficacy and prolonged duration of the treatment. According to WHO 2017 report [2], achieving high cure rate one of the key pillars of End TB strategy. The result of this study informs the NTPs to update the current treatment approaches as per recently recommended treatment guideline. However, prior to this prospective

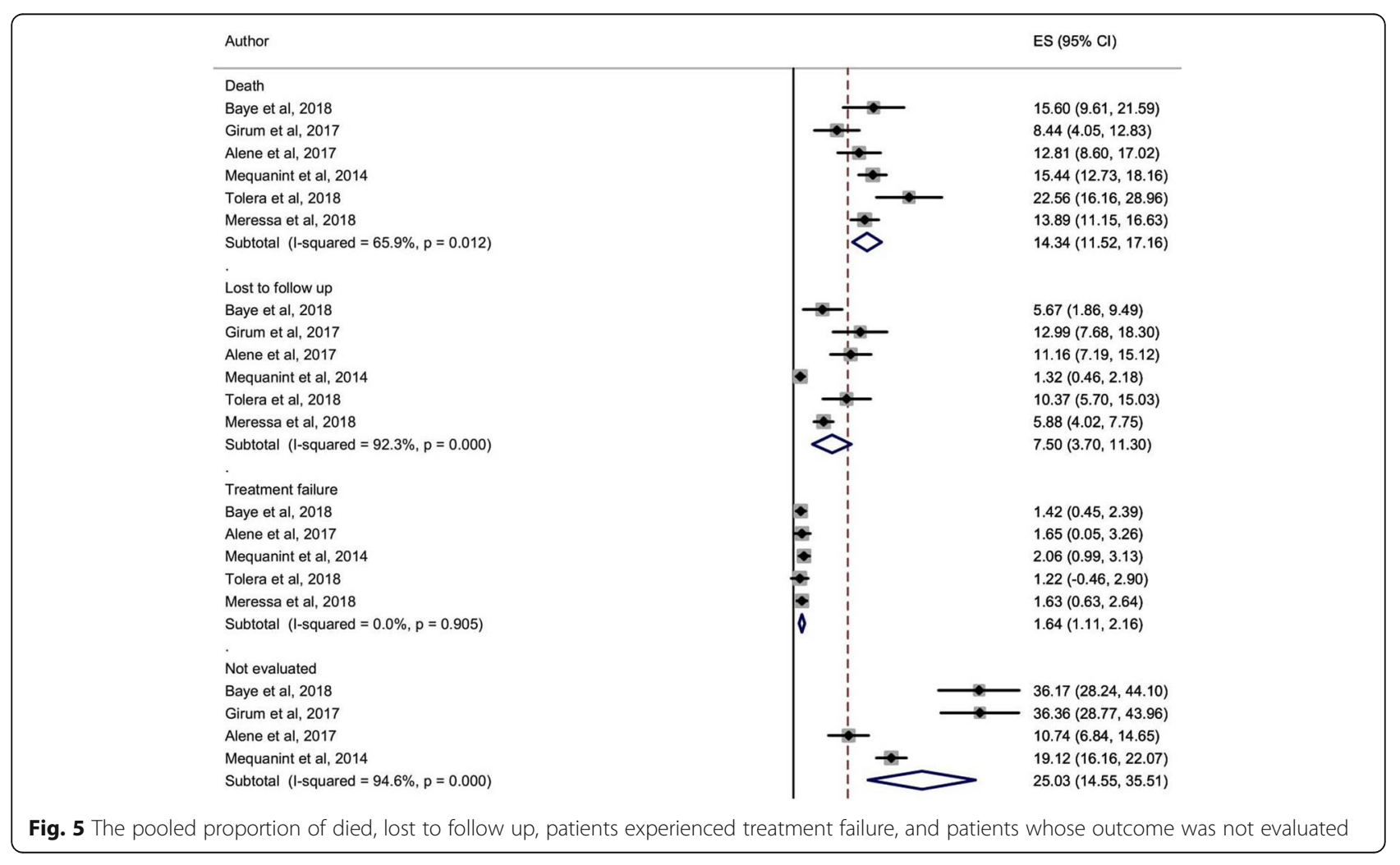


cohort analysis of a large number of patients required to support the evidence detailed above. Additionally, the Ethiopian Federal Ministry of Health should design smooth platforms to facilitate surveillance data on culture-based drug susceptibility testing; it may be used to identify a population of eligible patients for Short course MDR/RR-TB treatment.

\section{Limitations of the study}

One of the limitations of this analysis was observational and few studies have been included to measure MDR-TB treatment outcome. Potential predictors of poor treatment outcome Such as HIV infection and history TB treatment was not evaluated because the association was not measured in the original studies. Besides, sub-group analysis of the MDR-TB outcome was not done based on the mode of patient treatment (hospital and/or ambulatory) because lack of clear information in the reports.

\section{Conclusion}

The results suggest that low treatment success rate was estimated among MDR-TB patients whose composition of the treatment was based on a conventionally used treatment regimen. Therefore, our analysis remarks the need to test and implement recently adopted a shorter MDR-TB treatment regimen. Prior to implementation the levels of evidence need to be supported through rigorous institutional and national-wide studies.

\section{Additional files}

Additional file 1: Table S1. PRISMA 2009 checklist. (PDF 116 kb)

Additional file 2: Table S2. The quality assessment of the included studies into the present meta-analysis (DOCX $14 \mathrm{~kb}$ )

\section{Abbreviations}

$\mathrm{Cl}$ : Confidence interval; HIV: Human immuno-deficiency virus; MDRTB: Multidrug resistant Tuberculosis; NTPS: National Tuberculosis Control Programs; TB: Tuberculosis; WHO: World Health Organization

\section{Availability of data and materials}

All data generated or analyzed during this study are included in this published article [and its Additional file 1: Table S1 and Additional file 2: Table S2].

\section{Authors' contributions}

SE: Conception of study idea, study design, literature review, data collection, data extraction, data analysis and interpretation, and drafting manuscript. AA, FW, DG \& AF: Data collection and extraction, reviewing manuscript. US: Supervision, analysis and interpretation, and reviewing manuscript. All authors have read and approved the manuscript.

\section{Ethics approval and consent to participate} Not applicable.

\section{Consent for publication}

Not applicable.

\section{Competing interests}

The authors declare that they have no competing interests.

\section{Publisher's Note}

Springer Nature remains neutral with regard to jurisdictional claims in published maps and institutional affiliations.

\section{Author details}

${ }^{1}$ Department of Medical Microbiology, School of Biomedical and Laboratory Sciences, College of Medicine and Health Sciences, University of Gondar, Gondar, Ethiopia. ${ }^{2}$ Department of Clinical Nursing, College of Health Sciences, Debre Markos University, Debre Marqos, Ethiopia. ${ }^{3}$ Department of Immunology and Molecular Biology, College of Medicine and Health Sciences, University of Gondar, Gondar, Ethiopia. ${ }^{4}$ Department of Clinical Chemistry, College of Medicine and Health Sciences, University of Gondar, Gondar, Ethiopia. ${ }^{5}$ Institute of Clinical Immunology, Medical Faculty, University of Leipzig, Leipzig, Germany.

Received: 1 June 2018 Accepted: 20 September 2018

Published online: 27 September 2018

\section{References}

1. Adhvaryu M, Vakharia B. Drug-resistant tuberculosis: emerging treatment options. Clin Pharmacol. 2011;3:51-67.

2. World Health Organization. Global tuberculosis report 2017. Geneva: WHO; 2017.

3. World Health Organization. Global tuberculosis report. Geneva: WHO; 2016.

4. Eshetie S, Gizachew M, Dagnew M, Kumera G, Woldie H, Ambaw F, Tessema $B$, Moges F. Multidrug resistant tuberculosis in Ethiopian settings and its association with previous history of anti-tuberculosis treatment: a systematic review and meta-analysis. BMC Infect Dis. 2017;17(1):219.

5. Uplekar M, Weil D, Lonnroth K, Jaramillo E, Lienhardt C, Dias HM, Falzon D, Floyd K, Gargioni G, Getahun H. WHO's new end TB strategy. Lancet. 2015:385(9979):1799-801.

6. Khan FA, Salim MH, du Cros P, Casas EC, Khamraev A, Sikhondze W, Benedetti A, Bastos M, Lan Z, Jaramillo E. Effectiveness and safety of standardised shorter regimens for multidrug-resistant tuberculosis: individual patient data and aggregate data meta-analyses. Eur Respir J. 2017;50(1):1700061.

7. Bastos ML, Lan Z, Menzies D. An updated systematic review and metaanalysis for treatment of multidrug-resistant tuberculosis. Eur Respir J. 2017;49(3):1600803.

8. Fox GJ, Benedetti A, Cox H, Koh WJ, Viiklepp P, Ahuja S, Pasvol G, Menzies D. Group 5 drugs for multidrug-resistant tuberculosis: individual patient data. Eur Respir J. 2017:49(1):00993-2016.

9. Ahuja SD, Ashkin D, Avendano M, Banerjee R, Bauer M, Bayona JN, Becerra MC, Benedetti A, Burgos M, Centis R, et al. Multidrug resistant pulmonary tuberculosis treatment regimens and patient outcomes: an individual patient data meta-analysis of 9,153 patients. PLoS Med. 2012;9(8): e1001300.

10. Trebucq A, Schwoebel V, Kashongwe Z, Bakayoko A, Kuaban C, Noeske J, Hassane S, Souleymane B, Piubello A, Ciza F, et al. Treatment outcome with a short multidrug-resistant tuberculosis regimen in nine African countries. Int J Tuberc Lung Dis. 2018;22(1):17-25.

11. Aung KJ, Van Deun A, Declercq E, Sarker MR, Das PK, Hossain MA, Rieder HL. Successful '9-month Bangladesh regimen' for multidrug-resistant tuberculosis among over 500 consecutive patients. Int J Tuberc Lung Dis. 2014;18(10):1180-7.

12. Van Deun A, Maug AK, Salim MA, Das PK, Sarker MR, Daru P, Rieder HL. Short, highly effective, and inexpensive standardized treatment of multidrug-resistant tuberculosis. Am J Respir Crit Care Med. 2010;182(5):684-92.

13. Kuaban C, Noeske J, Rieder HL, Ait-Khaled N, Abena Foe JL, Trebucq A. High effectiveness of a 12-month regimen for MDR-TB patients in Cameroon. Int J Tuberc Lung Dis. 2015;19(5):517-24.

14. Piubello A, Harouna SH, Souleymane MB, Boukary I, Morou S, Daouda M, Hanki Y, Van Deun A. High cure rate with standardised short-course multidrug-resistant tuberculosis treatment in Niger: no relapses. Int J Tuberc Lung Dis. 2014;18(10):1188-94.

15. Falzon D, Schunemann HJ, Harausz E, Gonzalez-Angulo L, Lienhardt C, Jaramillo $\mathrm{E}$, Weyer $\mathrm{K}$. World health organization treatment guidelines for drug-resistant tuberculosis, 2016 update. Eur Respir J. 2017;49(3):1-12.

16. Federa Ministry of Health. Guidelines on programmatic management of drug resistant tuberculosis in Ethiopia. Addis, Ababa: MOH; 2013. 
17. Borenstein M, Hedges LV, Higgins JP, Rothstein HR. A basic introduction to fixed-effect and random-effects models for meta-analysis. Res Synth Methods. 2010;1(2):97-111.

18. Smith TC, Spiegelhalter DJ, Thomas A. Bayesian approaches to randomeffects meta-analysis: a comparative study. Stat Med. 1995;14(24):2685-99.

19. Rucker G, Schwarzer G, Carpenter JR, Schumacher M. Undue reliance on I(2) in assessing heterogeneity may mislead. BMC Med Res Methodol. 2008;8:79.

20. Baye AM, Sarhie WD, Endalew BT. Treatment outcomes of multi-drugresistant tuberculosis and its determinants in Boru Meda hospital, Northeast Ethiopia. J Infect Dis Ther. 2018;6(350):1-5.

21. Girum T, Tariku Y, Dessu S. Survival status and treatment outcome of multidrug resistant tuberculosis (MDR-TB) among patients treated in treatment initiation centers $(\mathrm{TIC})$ in South Ethiopia: a retrospective cohort study. Ann Med Health Sci Res. 2017;7:331-3.

22. Alene KA, Viney K, McBryde ES, Tsegaye AT, Clements AC. Treatment outcomes in patients with multidrug-resistant tuberculosis in north-West Ethiopia. Trop Med Int Health. 2017;22(3):351-62.

23. Mequanint G, Mengistu Z. Prevalence of MDR-TB and treatment outcome among tuberculosis patients attending at St. Peter TB Specialized Hospital. Addis Ababa: Addis Ababa University; 2014. Available at http://etd.aau.edu. et/bitstream/handle/123456789/7091/Gashaw\%20Mequanint.pdf?sequence= 1\&isAllowed $=\mathrm{y}$.

24. Tolera M, Dessie Y, Oljira L, Geleto A. Treatment outcome of multi drug resistant tuberculosis patients and its associated factors in Eastern Ethiopia. Ethiopia: Haramaya University; 2018.

25. Meressa D, Hurtado RM, Andrews JR, Diro E, Abato K, Daniel T, Prasad P, Prasad R, Fekade B, Tedla Y, et al. Achieving high treatment success for multidrug-resistant TB in Africa: initiation and scale-up of MDR TB care in Ethiopia--an observational cohort study. Thorax. 2015;70(12):1181-8.

26. World Health Organization. Towards universal access to diagnosis and treatment of multidrug-resistant and extensively drug-resistant tuberculosis by 2015: WHO progress report 2011. Geneva: WHO; 2011.

27. World Health Organization. The End TB Strategy. Geneva: WHO; 2017.

28. Federa Ministry of Health. Annual Tuberculosis Bulletin: Health Promotion and Diseases Prevention Directorate. Addis Ababa: $\mathrm{MOH} ; 2013$.

29. World Health Organization. Guidelines for the programmatic management of drug-resistant tuberculosis. Geneva: WHO; 2011.

30. Orenstein EW, Basu S, Shah NS, Andrews JR, Friedland GH, Moll AP, Gandhi NR, Galvani AP. Treatment outcomes among patients with multidrugresistant tuberculosis: systematic review and meta-analysis. Lancet Infect Dis, 2009;9(3):153-61.

31. Johnston JC, Shahidi NC, Sadatsafavi M, Fitzgerald JM. Treatment outcomes of multidrug-resistant tuberculosis: a systematic review and meta-analysis. PLoS One. 2009:4(9):e6914.

Ready to submit your research? Choose BMC and benefit from:

- fast, convenient online submission

- thorough peer review by experienced researchers in your field

- rapid publication on acceptance

- support for research data, including large and complex data types

- gold Open Access which fosters wider collaboration and increased citations

- maximum visibility for your research: over $100 \mathrm{M}$ website views per year

At $\mathrm{BMC}$, research is always in progress.

Learn more biomedcentral.com/submissions 